\title{
A produção do conhecimento em Educação Física e suas subáreas: um panorama a partir de periódicos nacionais da área
}

\author{
Scientific publications in physical education and related areas: a snapshot \\ based on brazilian journals in the field
}

Marluce Raquel Decian Corrêa', Eduardo Lucia Caputo', Fernanda Stein', Priscila Lopes Cardozo', Helena Thofehrn Lessa', Rodrigo Kohn Cardoso', Marlos Rodrigues Domingues', Pedro Curi Hallal'

\section{Resumo}

Esse artigo descreve a produção do conhecimento em Educação Física em periódicos brasileiros da área, agrupando esses produtos em subáreas, verificando sua relação com a Pós-Graduação e a distribuição geográfica dos autores com base na sua afiliação institucional, a partir do último número disponível de cada periódico. Trata-se de análise documental, com dados extraídos do website dos 12 periódicos científicos da Educação Física classificados nos estratos B2, ou superior do WebQualis da CAPES de 2014. O último número disponível de cada periódico foi avaliado e os artigos publicados foram categorizados em subáreas, descritas a seguir. Foram analisados 163 artigos relacionados à área da Educação Física, distribuídos nas seguintes subáreas: Pedagógica e Sociocultural $=42,9 \%$, Treinamento Físico e Fisiologia $=26,5 \%$, Atividade Física e Saúde $=20,4 \%$, Comportamento Motor $=10,2 \%$. Pelo menos um autor dos artigos estava vinculado a um Programa de Pós-Graduação em Educação Física em 99 (60,7\%) dessas publicações. A maioria das publicações são oriundas de programas com conceito quatro e cinco da CAPES. Assinala-se a importância de os Programas de Pós-Graduação com conceitos superiores a quatro publicarem com maior frequência em revistas brasileiras, a fim de fortalecer os periódicos nacionais e auxiliar na consolidação da área da Educação Física no país.

\section{Palavras-chave}

Pesquisa; Publicações periódicas; Artigo de revista; Política de educação superior.

\begin{abstract}
This article described publications related with Physical Education in Brazilian scientific journals, grouped publications by sub-area and verified its connection with specific graduation programs and geographical distribution of authors' institutional affiliation based on the last available number of each periodical. Data were extracted from the website of 12 scientific journals that publish Physical Education related articles and are classified as $B 2$ or higher in the WebQualis system proposed by CAPES in 2014. The most recent issue of each journal was evaluated and the articles were classified into sub-areas described below. We analyzed 163 articles related with Physical Education distributed in the following subareas: Pedagogic and Sociocultural $=49.2 \%$, Physical Training and Physio$\log y=26.5 \%$, Physical Activity and Health $=20.4 \%$, Motor Behavior $=10.2 \%$. In 99 publications (60.7\%) at least one author was a postgraduate program member. Most publications were from postgraduate programs with scores of four or five (scores range from three to seven). In order to strengthen national journals and help to consolidate the area of Physical Education in the country we emphasize the importance of post-graduation programs with concepts above 4 to publish more frequently in Brazilian journals.
\end{abstract}

\section{Keywords}

Research; Periodicals; Journal article; Higher education policy.

\section{Introdução}

A produção do conhecimento em Educação Física tem aumentado simultaneamente ao processo histórico de consolidação da área no país ${ }^{1}$. Percebe-se que sua intensificação se deu a partir da inserção e expan-

1 Escola Superior de Educação Física, Universidade Federal de Pelotas, Pelotas, RS são dos cursos de Pós-Graduação Stricto Sensu no país, havendo aumento significativo principalmente na última década ${ }^{2,3}$. Em 1980, a área contava com dois cursos de mestrado e nenhum de doutorado. Uma década depois, estes números mudaram para sete e um; em 2000 eram doze e sete; e em 2010, existiam 21 cursos de mestrado e nove de doutorado no país ${ }^{4}$. 
De acordo com dados disponibilizados pela Coordenação de Aperfeiçoamento de Pessoal de Nível Superior - CAPES 5 , existem 32 cursos de mestrado e 19 cursos de doutorado. Associado a este aumento no número de cursos de Pós-Graduação, a produção científica na área passou a apresentar números também mais elevados ${ }^{3}$.

Os Programas de Pós-Graduação da área da Educação Física, de forma geral, estão configurados através de duas principais áreas de concentração, as Ciências Sociais e Humanas e as Ciências Biológicas e da Saúde e, a partir delas, estruturam-se as linhas de pesquisas. De acordo com o modelo proposto por Tani ${ }^{6}$, a Cinesiologia (termo que significa "estudo do movimento" e é adotado pelo autor para denominar a área da Educação Física, a qual, segundo ele, tem sua identidade acadêmica facilitada por meio dessa identificação), segue uma organização transdisciplinar, a qual é composta por três principais subáreas de investigação: Biodinâmica do Movimento Humano, Comportamento Motor e Humano e Estudos Socioculturais do Movimento Humano. A subárea Biodinâmica do Movimento Humano é constituída pela Bioquímica do Exercício, Fisiologia do Exercício, Biomecânica e Cineantropometria. Já a subárea Comportamento Motor e Humano engloba o Controle Motor, Aprendizagem Motora, Desenvolvimento Motor e Psicologia do Esporte e, por fim, a subárea Estudos Socioculturais do Movimento Humano é composta pela Sociologia, História, Antropologia, Filosofia, Ética e Estética do Movimento Humano/Esporte.

Partindo dessa estruturação e das atuais linhas de pesquisa dos Programas de Pós-Graduação em Educação Física no Brasil, procurou-se adaptar o modelo sugerido por Tani ${ }^{6}$, uma vez que a Atividade Física e Saúde parece não se enquadrar nas respectivas subáreas. A Atividade Física e Saúde é uma linha de investigação em evidência e que, de acordo com dados disponibilizados pela Plataforma Sucupira da CAPES 7 , está contemplada em 77,8\% dos Programas de Pós-Graduação em Educação Física no Brasil. Sendo assim, a fim de compor todas as subáreas mencionadas anteriormente, as mesmas foram assim agrupadas: (1) Pedagógica e Sociocultural; (2) Treinamento Físico e Fisiologia; (3) Comportamento Motor; (4) Atividade Física e Saúde.

Considerando o fenômeno de expansão da Educação Física, presente tanto no número de cursos de Pós-Graduação quanto no número de artigos publicados, e a evidente organização da sua área de conhecimento em subáreas de pesquisa, torna-se importante conhecer o rumo das publicações realizadas no país e refletir sobre as práticas desta área específica, buscando apontar possíveis lacunas e direções nas investigações. Nesse sentido, o presente artigo se propõe a descrever a produção do conhecimento em Educação Física em periódicos brasileiros da área, agrupando esses produtos em subáreas, verificando sua relação com a Pós-Graduação e a distribuição geográfica dos autores com base na sua afiliação institucional, a partir do último número disponível de cada periódico.

\section{Métodos}

Esse estudo é caracterizado como descritivo/exploratório, recorrendo à técnica de análise documental, que se utiliza de materiais que ainda não receberam tratamento analítico ${ }^{8}$. Para realizar o panorama da produção de conhecimento na Educação Física em periódicos brasileiros, extraíram-se os dados a partir de busca no sistema de periódicos WebQualis da CAPES na área de avaliação em Educação Física no ano de 2014, a partir dos estratos B2 ou superior. A coleta e a extração dos dados foram realizadas por dois pesquisadores de forma independente. A seleção dos periódicos foi realizada inicialmente pela leitura dos seus títulos. Foram 
incluídos aqueles cujos títulos se encontravam na língua portuguesa e/ou continham o termo em inglês "Brazilian".

Na sequência, a delimitação foi realizada de acordo com a presença das seguintes palavras-chave em seus títulos: saúde, esporte, movimento e educação física. Além disso, os escopos das revistas foram verificados, a fim de identificar se os periódicos poderiam ser caracterizados e incluídos dentro da área de interesse do estudo. Doze periódicos foram selecionados para análise: Motriz: Revista de Educação Física, Revista Brasileira de Medicina do Esporte, Revista Movimento, Revista Brasileira de Cineantropometria e Desempenho Humano, Revista da Educação Física/UEM, Revista Brasileira de Educação Física e Esporte, Revista Brasileira de Ciências do Esporte, Licere, Pensar a Prática, Revista Brasileira de Biomecânica, Revista Brasileira de Ciência e Movimento e Revista Brasileira de Atividade Física e Saúde. Para melhor compreensão dos periódicos incluídos, ver fluxograma (Figura 1). Embora a Licere trate de um periódico que se dedica a discutir a temática Lazer a partir de uma ótica multidisciplinar, Isayama e Melo apontam que a maior parte dos autores dos artigos publicados no periódico são oriundos da área de conhecimento da Educação Física, bem como seu corpo editorial. Ademais, os autores afirmam o quanto a trajetória do periódico Licere tem relação com o trato do seu tema central no âmbito das iniciativas relacionadas à Educação Física.

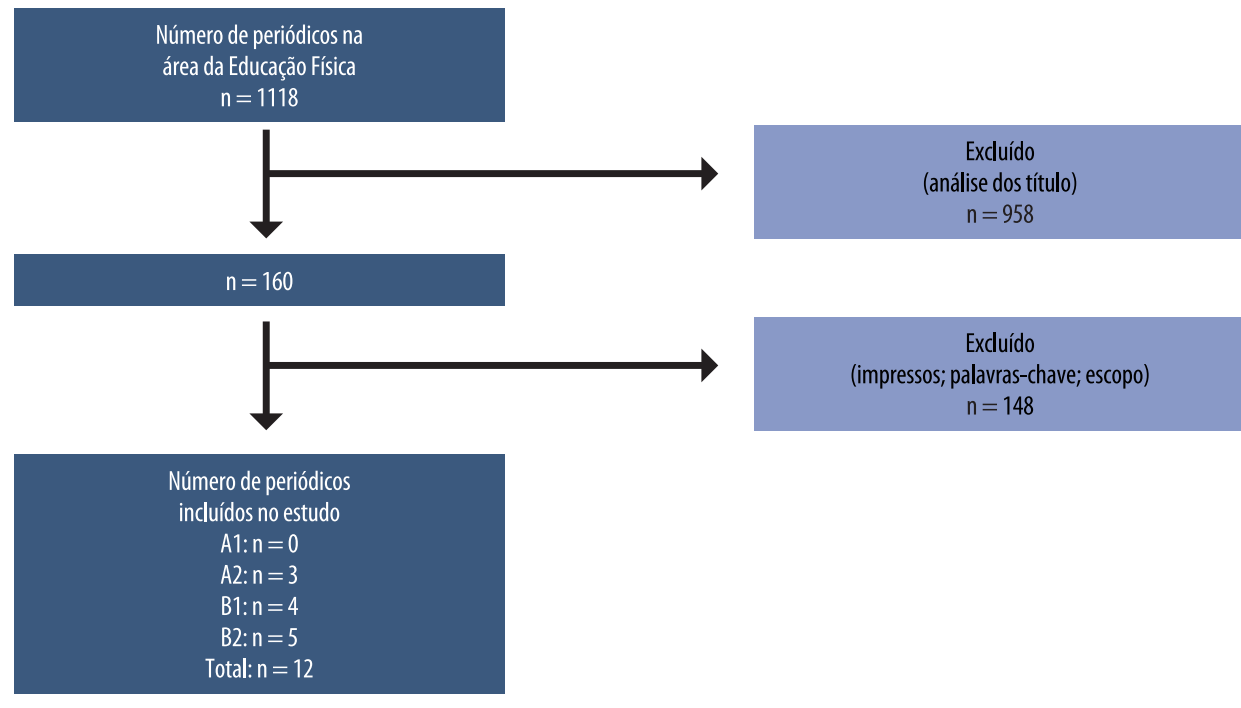

FIGURA 1 - Fluxograma da seleção dos Periódicos Nacionais da Educação Física incluídos no estudo.

Os sítios dos periódicos foram visitados pelos pesquisadores no dia 24/03/2015, a fim de verificar o conteúdo do último número publicado, com exceção de números relacionados a edições especiais. Neste caso, o número imediatamente anterior ao mesmo foi selecionado. Os artigos publicados foram divididos em subáreas: (1) Pedagógica e Sociocultural; (2) Treinamento Físico e Fisiologia; (3) Comportamento Motor; (4) Atividade Física e Saúde. Sendo que, a Pedagógica e Sociocultural orienta-se epistemologicamente nas ciências sociais e humanas, o Comportamento Motor foca nos processos neuropsicológicos da organização motora em termos de controle, desenvolvimento e aprendizagem motora, enquanto que o "Treinamento Físico e Fisiologia”, "Atividade Física e Saúde” baseiam-se nos princípios da biodinâmica do movimento humano.

Para classificar os artigos nas respectivas subáreas, as publicações foram analisadas de forma aleatória por dois pesquisadores. Caso houvesse divergências, eles procuravam estabelecer um consenso e, se as mesmas permaneciam, um terceiro 
pesquisador era consultado. A identificação dos manuscritos nas subáreas se deu por meio da análise dos títulos, palavras-chave e resumo. Os artigos foram lidos na íntegra quando não tinham informações suficientes para classificação dos mesmos nas subáreas.

Para estabelecer relações com os autores das publicações, algumas informações foram extraídas, tais como: (1) identificar em quais universidades eles eram vinculados e se, pelo menos, um autor de cada artigo estava credenciado em algum Programa de Pós-Graduação em Educação Física. Foi utilizado como critério, verificar se o primeiro autor possuía vínculo com algum programa; caso o mesmo não fosse credenciado, passava-se para o segundo autor e assim sucessivamente; (2) A partir dessa seleção, também foi verificada a relação entre os autores incluídos e os conceitos dos programas estabelecidos pela CAPES; (3) Visitas aos sítios dos Programas de Pós-Graduação de Educação Física a fim de identificar suas linhas de pesquisa.

\section{Resultados}

A partir da análise por estrato na área Educação Física do sistema WebQualis do portal da CAPES, um total de 1.118 periódicos foram encontrados. Após a aplicação dos critérios de exclusão, 12 periódicos foram incluídos no estudo. Destes, três eram classificados como A2 (Motriz, Movimento, Revista Brasileira de Medicina do Esporte), quatro como B1 (Revista Brasileira de Ciência do Esporte, Revista Brasileira de Cineantropometria e Desempenho Humano, Revista Brasileira de Educação Física e Esporte, Revista da UEM) e cinco como B2 (Licere, Pensar a Prática, Revista Brasileira de Atividade Física e Saúde, Revista Brasileira de Biomecânica, Revista Brasileira de Ciência e Movimento). A periodicidade dos volumes das revistas incluídas variou de bimestral a quadrimestral, e no mínimo sete e no máximo 15 artigos originais incluídos no volume analisado, conforme exposto na tabela 1.

TABELA 1 - Descrição dos periódicos analisados.

\begin{tabular}{|c|c|c|c|c|}
\hline Revista & Periodicidade & Volume & Número & $\begin{array}{l}\text { Número de artigos originais } \\
\qquad(n)\end{array}$ \\
\hline Motriz: Revista de Educação Física & Trimestral & 21 & 1 & 13 \\
\hline Revista Brasileira de Medicina do Esporte & Bimestral & 21 & 2 & 15 \\
\hline Revista movimento & Trimestral & 21 & 1 & 12 \\
\hline Cineantropometria e Desempenho Humano & Bimestral & 17 & 2 & 12 \\
\hline Revista da Educação Física/UEM & Trimestral & 26 & 1 & 13 \\
\hline Revista Brasileira de Educação Física e Esporte & Trimestral & 29 & 1 & 13 \\
\hline Revista Brasileira de Ciências do Esporte & Trimestral & 37 & 1 & 12 \\
\hline Licere & Trimestral & 18 & 1 & 10 \\
\hline Pensar a Prática & Trimestral & 18 & 1 & 14 \\
\hline Revista Brasileira de Biomecânica & Quadrimestral & 16 & 33 & 7 \\
\hline Revista Brasileira de Ciência e Movimento & Trimestral & 23 & 1 & 15 \\
\hline Revista Brasileira de Atividade Física e Saúde & Bimestral & 20 & 2 & 7 \\
\hline
\end{tabular}

Nesses periódicos, foi publicado um total de 163 artigos relacionados à Educação Física, distribuídos nas seguintes subáreas: Pedagógica e Sociocultural = 42,9\%, Treinamento Físico e Fisiologia = 26,5\%, Atividade Física e Saúde = 20,4\%, Comportamento Motor 10,2\%. 
A Tabela 2 descreve as subáreas dos artigos de acordo com sua classificação no WebQualis. As subáreas Pedagógica e Sociocultural (46,9\%) e Atividade Física e Saúde $(42,3 \%)$ concentram suas publicações nos periódicos do estrato B2, enquanto as subáreas de Treinamento Físico/Fisiologia (39,6\%) e Comportamento Motor $(57,2 \%)$ concentram suas produções nos periódicos do estrato B1.

TABELA 2 - Número e percentual de artigos publicados em periódicos nacionais pelas diferentes subáreas do conhecimento da Educação Física em cada estrato de classificação dos periódicos.

\begin{tabular}{lcccccc}
\hline Estrato & $\begin{array}{c}\text { Pedagogia } \\
\text { Socioculturais } \\
\mathrm{n}(\%)\end{array}$ & $\begin{array}{c}\text { Treinamento } \\
\text { Fisiologia } \\
\mathrm{n}(\%)\end{array}$ & $\begin{array}{c}\text { Comportamento } \\
\text { Motor } \\
\mathrm{n}(\%)\end{array}$ & $\begin{array}{c}\text { Atividade Física } \\
\text { e Saúde } \\
\mathrm{n}(\%)\end{array}$ & $\begin{array}{c}\text { Outras } \\
\text { áreas } \\
\mathrm{n}(\%)\end{array}$ & $\begin{array}{c}\text { Total } \\
\mathrm{n}(\%)\end{array}$ \\
\hline A1 & - & - & - & - & - & - \\
\hline A2 & $18(28,1)$ & $14(29,1)$ & $1(7,1)$ & $9(24,3)$ & $3(23,1)$ & $45(25,6)$ \\
\hline B1 & $16(25,0)$ & $19(39,6)$ & $8(57,2)$ & $12(32,4)$ & $1(7,7)$ & $56(31,8)$ \\
\hline B2 & $30(46,9)$ & $15(31,3)$ & $5(35,7)$ & $16(43,3)$ & $9(69,2)$ & $75(42,6)$ \\
\hline Total & $64(100,0)$ & $48(100,0)$ & $14(100,0)$ & $37(100,0)$ & $13(100,0)$ & $176(100,0)$ \\
\hline
\end{tabular}

Foi encontrado pelo menos um autor vinculado a algum Programa de Pós-Graduação em Educação Física em 99 (60,7\%) das publicações. Com relação à distribuição geográfica, a maioria dos autores se concentram nas regiões Sudeste $(42,7 \%)$ e Sul $(34,6 \%)$ e subsequentemente nas regiões Nordeste $(12,4 \%)$, Centro-Oeste $(9,5 \%)$ e Norte $(0,8 \%)$. Com relação ao número de autores por publicação, cada artigo contou, em média, com 4,1 autores.

De forma geral, os artigos somaram 672 autores. Em relação ao percentual de autores em cada subárea, observou-se uma proporção maior na subárea Fisiologia $(34,8 \%)$, seguido pela Pedagogia (27,4\%), Atividade Física (26.8\%) e Comportamento Motor (11,0\%).

A Tabela 3 apresenta a relação entre o estrato dos periódicos em que os artigos foram publicados e o conceito do PPG no qual o primeiro autor está vinculado. Dos programas de conceito três ou cinco, a maior parte dos produtos foi publicado no estrato B2. Nos programas de conceito quatro ou sete, a maior parte dos produtos foi publicada em periódicos de conceito B1. Apenas no conceito seis, a maior parte das produções se deu no estrato A2.

TABELA 3 - Relação entre o conceito dos Programas de Pós-Graduação na área de Educação Física e o número de publicações em cada estrato dos periódicos.

\begin{tabular}{lccccc}
\hline \multicolumn{7}{c}{ Conceitos dos Programas de Pós-Graduação (2015) } \\
\hline Estrato & 3 & 4 & 5 & 6 & 7 \\
\hline A1 & - & - & - & - & - \\
\hline A2 & 5 & 7 & 6 & 4 & 2 \\
\hline B1 & 3 & 19 & 11 & 1 & 4 \\
\hline B2 & 6 & 17 & 13 & 1 & - \\
\hline Total & 14 & 43 & 30 & 6 & 6 \\
\hline
\end{tabular}

A tabela 4 estabelece uma relação entre a quantidade de linhas de pesquisa nos Programas de Pós-Graduação da área de Educação Física e os produtos publicados nos periódicos avaliados. Foram identificadas 48 linhas de pesquisa na subárea Pedagógica e Sociocultural; 47 no Treinamento Físico e Fisiologia; 38 na Atividade Física e Saúde; e 17 no Comportamento Motor. As subáreas com maior número de linhas de pesquisa foram exatamente aquelas com maior volume de publicações nos periódicos analisados. 
TABELA 4 - Número de publicações por subárea e número de Programas de Pós-Graduação (PPG's) na área de Educação Física que possuem linhas referentes a essas subáreas.

\begin{tabular}{lcc}
\hline Subárea & Publicações & PPG's \\
\hline Pedagogia e Socioculturais & 64 & 48 \\
\hline Fisiologia e Treinamento & 48 & 47 \\
\hline Comportamento Motor & 14 & 17 \\
\hline Atividade Física e Saúde & 37 & 38 \\
\hline Total & 163 & 150 \\
\hline
\end{tabular}

\section{Discussão}

Com base nos percentuais dos artigos publicados pelas diferentes subáreas do conhecimento da Educação Física, foi possível verificar que a subárea Pedagógica e Sociocultural apresenta o maior volume de manuscritos, quando comparada às demais, podendo-se inferir que esta subárea concentra suas publicações em periódicos nacionais da área de Educação Física. Os achados ainda mostram que essa subárea é a que mais produz no estrato A2. Logo, pode-se dizer que isso ocorre em virtude de um periódico específico (Movimento) ter em seu escopo o objetivo de divulgar a produção científica de temas relacionados à Educação Física em interface com as Ciências Humanas e Sociais, no que diz respeito aos seus aspectos pedagógicos, históricos, políticos e culturais. Outra característica peculiar é o hábito dos pesquisadores desta subárea eleger periódicos nacionais e não internacionais para divulgação de seus produtos. Este aspecto também pode estar atribuído ao número restrito de periódicos classificados no WebQualis CAPES com escopo direcionado para esta subárea ${ }^{10}$.

A partir da reforma curricular de $1987^{10}$ que os aspectos sócio-filosóficos da formação geral passam a ser consubstanciados em três áreas do conhecimento: conhecimentos filosóficos, conhecimentos do ser humano e conhecimentos da sociedade. Por conseguinte, mudanças parecem estar refletindo na pesquisa brasileira em Educação Física, através dos periódicos analisados. Diferentemente, o estudo realizado por Rosa e Leta ${ }^{11}$ aponta que publicações com enfoque em áreas mais humanísticas e/ou sociais, como a Filosofia, a História, a Sociologia do Esporte, a Psicologia aplicada à Educação Física e ao esporte, a Didática, são encontradas em minoria nos periódicos nacionais. No entanto, tal achado pode estar relacionado ao presente estudo ter se limitado apenas ao último número dos periódicos nacionais da Educação Física, restringindo o delineamento preciso do perfil da publicação na área. Outro aspecto que pode explicar fortemente essa discordância é o de que o estudo de Rosa e Leta ${ }^{11}$ não incluiu a Revista Movimento na análise da produção científica. Ainda, tal realidade apontada pelos autores pode ter sido alterada nos últimos anos, visto que a pesquisa citada foi realizada no ano de 2007.

No que tange às publicações nos estratos $\mathrm{B} 1$, a subárea que apresenta maior produtividade é a de Treinamento Físico e Fisiologia, o que pode ser explicado pela grande quantidade de linhas de pesquisa nos programas de Pós-graduação em Educação Física no Brasil que estudam essa temática específica. Há uma relação direta entre linhas de pesquisa e número de publicações. Esse dado vai ao encontro dos resultados demonstrados por Rosa e Leta ${ }^{11}$, que encontraram maior publicação também na subárea da Fisiologia.

A subárea de Comportamento Motor é a que menos publica em todos os estratos, apresentando índice maior no estrato B2. Deduz-se que isso ocorra pelo fato dessa subárea apresentar inferioridade numérica nas linhas de pesquisa dos programas de pós-graduação em Educação Física no país e, ainda, por possuir a 
tradição de publicação em periódicos internacionais.

No que se refere ao percentual de número de autores por região, identificou-se que as regiões brasileiras com maior número de autores são, respectivamente, região Sudeste (42,7\%), região Sul (34,6\%), região Nordeste (12,4 \%), região Centro-Oeste $(9,5 \%)$ e região Norte $(0,8 \%)$. Tal distribuição, com ênfase na região Sudeste e Sul, pode ser entendia devido à concentração de maior número de universidades e Programas de Pós-Graduação na área de Educação Física nessas regiões, bem como pelos maiores investimentos, tanto de infraestrutura quanto de recursos humanos nas regiões Sul e Sudeste ${ }^{12}$.

Quanto ao número de autores por artigos, a média geral foi de 4,1. No entanto, se isolarmos a subárea Pedagógica e Sociocultural, essa média cai para 2,9, constituindo a subárea que apresenta em média a menor quantidade de autores por artigo. Isso representa uma peculiaridade da área por se tratar, na maioria das vezes, de estudos densos, com grande aprofundamento teórico, além de seus instrumentos para a coleta e interpretação dos dados serem, principalmente, de cunho qualitativo. Nas outras subáreas, a média foi próxima de cinco autores por artigo.

Os Programas de Pós-Graduação em Educação Física com conceitos seis e sete sinalizaram menor quantidade de publicações em revistas nacionais. Tal indicativo pode estar atribuído aos programas de conceito mais elevado enviarem seus manuscritos a periódicos internacionais e com maior fator de impacto, permitindo maior visibilidade. Enquanto isso, os programas com conceito quatro e cinco encontram-se entre os que mais publicam nos periódicos nacionais e, principalmente, nos estratos B1 e B2. Esses dados podem estar relacionados com a intenção desses programas de se manterem ou aumentarem seu conceito a partir do número de publicações. Já os programas com conceito três apresentam número menor de publicações, possivelmente porque se configuram como programas recentemente credenciados com produções em fase de crescimento, ou ainda programas com indícios de descredenciamento.

Na relação entre a estratificação dos periódicos e os Programas de Pós-Graduação da área da Educação Física, esperava-se que os programas com conceito sete teriam número elevado de publicações em revistas de estrato A2, em comparação aos demais programas com conceitos mais baixos, no entanto, apenas dois artigos, de um total de seis, foram publicados por autores vinculados a programas com conceito sete. Estima-se que os autores vinculados a tais programas publicam com maior frequência em periódicos internacionais, já que o formato de avaliação dos Programas de Pós-Graduação exige a necessidade de internacionalização das publicações, principalmente em periódicos com alto Fator de Impacto. Esse fato também foi identificado por Job, Fraga e $\mathrm{Neto}^{13}$, como consequência da forma de análise de medidas bibliométricas pelas quais as revistas científicas passam: padrões internacionais para mensuração de produção nacional; estabelecer comparações de áreas diferentes; comparação entre países com características socioeconômicas diferentes. Em consequência disso, os pesquisadores, principalmente da subárea biomédica, preferem enviam seus manuscritos a periódicos indexados nas bases de dados de maior notoriedade, em detrimento dos periódicos nacionais ${ }^{13}$.

De forma geral, percebe-se um movimento nacional de valorização da subárea Pedagógica e Sociocultural. Este fato permite repensar a questão dessa subárea publicar em periódicos nacionais na área de Educação Física. Faz-se necessário para a Educação Física brasileira que tais publicações aconteçam tanto em nível nacional quanto internacional, visando difusão em maior escala do conhecimento produzido nesta subárea, assim como sua maior valorização e visualização dos percursos 
científicos como um todo. Existem algumas possibilidades de periódicos internacionais e com estratificação elevada que contemplam a subárea Sociocultural e Pedagógica, quais sejam: Sport Education and Society; Journal of Teaching in Physical Education; European Journal of Sport Science; Journal of the Philosophy of Sport; Cultura, Ciencia y Deporte; Leisure Studies; Loisir et Société/Society \& Leisure; Physical eEucation and Sport Pedagogy, dentre outros.

Assinala-se a importância de os Programas de Pós-Graduação com conceitos superiores a quatro publicarem com maior frequência em revistas brasileiras, a fim de fortalecer os periódicos nacionais e auxiliar na consolidação da área da Educação Física no país.

Percebe-se ainda, que justamente devido à publicação no exterior em revistas de maior visibilidade é que alguns Programas de Pós-Graduação apresentam conceitos elevados. Uma vez atingido tal objetivo através da publicação em periódicos internacionais, estes cursos assumem maior responsabilidade e capacidade de fortalecimento da pesquisa nacional, sendo um dos caminhos o aumento da publicação em periódicos brasileiros.

Embora o estudo aborde questões pertinentes à área, algumas limitações precisam ser destacadas. A principal limitação foi em relação à coleta de dados, a qual foi realizada apenas no último número publicado em periódicos nacionais. Outra limitação foi ter incluído apenas periódicos nacionais, o que dificultou a elaboração de algumas inferências acerca das subáreas e suas preferências na escolha dos periódicos, limitando as generalizações dos achados.

Contudo, possíveis futuros estudos poderão apresentar o quadro nacional completo e comparações longitudinais. Por fim, espera-se que esse estudo possa servir de apoio para demais pesquisadores com interesse na área.

Além disso, sugere-se que novos estudos sejam realizados estendendo as análises a mais números de cada periódico e incluindo periódicos internacionais a fim de ampliar as discussões do tema.

\section{Colaboração dos autores}

M. R. Domingues (0000-0002-2503-2944), P. C. Hallal (0000-0003-1470-6461) idealizaram o estudo e supervisionaram todas as etapas do trabalho. M. R. D. Corrêa (00000002-6920-2925), E. L. Caputo (0000-0002-3515-9308), F. Stein (0000-0002-79389703), P. L. Cardozo (0000-0002-5996-6868), H. T. Lessa (000-0003-2099-7701), R. K. Cardoso (000-0002-0914-1251), trabalharam no planejamento, na coleta de dados e na redação do manuscrito, dando contribuições intelectuais para o aprimoramento do mesmo. Todos os autores aprovaram a versão submetida para publicação.

\section{Conflito de interesse}

Os autores declaram não haver conflitos de interesse.

\section{Referências}

1. Coutinho RX, Soares MC, Folmer V, Puntel RL. Análise da produção de conhecimento da Educação Física brasileira sobre o cotidiano escolar. Rev Bras Pós Grad. 2012;9(17):491-516.

2. Quadros HM, Afonso, MR, Ribeiro, JAB. O cenário da pós-graduação em Educação Física: contextos e possibilidades na região sul do Brasil. Rev Bras Ativ Fis Saude. 2013;18(5):576-84.

3. Tani G. Os desafios da pós-graduação em Educação Física. Rev Bras Cienc Esporte. 2000;22(1):79-90.

4. Rigo LC, Ribeiro GM, Hallal, PC. Unidade na diversidade: desafios para a Educação Física no século XXI. Rev Bras Ativ Fis Saude. 2011;16(4):339-45. 
5. Coordenação de Aperfeiçoamento de Pessoal de Nível Superior (CAPES). Cursos recomendados. <acesso em 26 de mar, 2015> https://sucupira.capes.gov.br/sucupira/ public/consultas/coleta/programa/quantitativos/quantitativoAreaAvaliacao. jsf;jsessionid=LGzmWH42EWxm0SBhtmhLYDlY.sucupira-203

6. Tani G. Leituras em Educação Física: retratos de uma jornada. São Paulo: Phorte, 2011.

7. Plataforma Sucupira. Relatórios de Dados de Envio do Coleta. <acesso em 27 de dez, 2016> https://sucupira.capes.gov.br/sucupira/public/informacoes_programa/ informacoesPrograma.jsf

8. Gil, AC. Métodos e técnicas de pesquisa social. São Paulo: Atlas, 2010.

9. Isayama HF, Melo VA. Licere: uma revista brasileira de lazer. Rev Bras Ciênc Esporte. 2013;36(4):773-79.

10. Oliveira VM. Consenso e conflito da educação física brasileira. São Paulo: Papirus, 1994.

11. Rosa S, Leta J. Tendências atuais da pesquisa brasileira em Educação Física Parte 1: uma análise a partir de periódicos nacionais. Rev Bras Educ Fís Esporte. 2010;24(1):121-34.

12. Manoel E, Carvalho Y. Pós-graduação na Educação Física brasileira: a atração (fatal) para a biodinâmica. Educação e Pesquisa. 2011;37(2):389-406.

13. Job I, Fraga AB, Neto VM. Invisibilidade das revistas científicas brasileiras de educação física nas bases de dados. Cadernos Bad. 2008;1:15-26.

Endereço para

Correspondência

Nome

email@email.com
Escola Superior de Educação Física da

Universidade Federal de Pelotas

Rua Luís de Camões, 625. Três Vendas,

Cep: 96055-630. Pelotas - RS, Brasil.

Fone: (53) 3273-2752
Recebido

Revisado

$08 / 09 / 2016$

$05 / 12 / 2016$

$09 / 01 / 2017$

$15 / 02 / 2017$

Aprovado $\quad 17 / 02 / 2017$ 